See Article page 128.

\section{Commentary: Hepatocellular carcinoma: A threat for patients with Fontan circulation}

\author{
Juan Contreras, MD, and \\ David Faraoni, MD, PhD, FAHA ${ }^{\mathrm{b}}$
}

Since the first Fontan operation was performed in 1968, several modifications have been introduced that led to improvement in short- and long-term survival. The current 30 -year survival after Fontan completion is $85 \%$. ${ }^{1,2}$ Hemodynamic changes associated with the Fontan circulation, including elevated central venous pressure and diminished cardiac output, as well as chronic hypoxic injury of the liver, are responsible for the development of Fontan-associated liver disease (FALD). ${ }^{1}$ In Fontan circulation, the hepatic veins discharge directly into the Fontan circuit and the liver is therefore particularly susceptible to the effects of central venous hypertension. The elevated central venous pressure produces a centrilobular hepatic congestion and necrosis, and activation of inflammatory mediators that perpetuate the chronic inflammation and subsequent fibrosis of the liver. $^{3,4}$ The pathophysiological mechanisms promoting liver injury and fibrosis in FALD are summarized in Figure 1.

Histological studies suggest that $43 \%$ of patients with Fontan circulation have evidence of advanced liver fibrosis 30 years after the Fontan completion. ${ }^{5}$ Liver fibrosis appears to be an inevitable complication in the Fontan circulation and the degree of fibrosis is increasing over time. In a retrospective study of Fontan patients receiving surveillance for FALD, 62\% had evidence of chronic liver

\footnotetext{
From the ${ }^{\mathrm{a}}$ Department of Cardiovascular Surgery and ${ }^{\mathrm{b}}$ Division of Cardiac Anesthesia, Department of Anesthesiology and Pain Medicine, The Hospital for Sick Children, University of Toronto, Toronto, Ontario, Canada

Disclosures: The authors reported no conflicts of interest.

The Journal policy requires editors and reviewers to disclose conflicts of interest and to decline handling or reviewing manuscripts for which they may have a conflict of interest. The editors and reviewers of this article have no conflicts of interest.

Received for publication Feb 5, 2020; revisions received Feb 5, 2020; accepted for publication Feb 20, 2020; available ahead of print April 3, 2020.

Address for reprints: David Faraoni, MD, PhD, FAHA, Division of Cardiac Anesthesia, Department of Anesthesiology and Pain Medicine, Hospital for Sick Children, 555 University Ave, Black Wing 2nd Floor, Room 2420, Toronto, Ontario M5G 1X8 Canada (E-mail: david.faraoni@sickkids.ca).

JTCVS Techniques 2020;2:131-2

2666-2507

Copyright $(\underset{2}{ } 2020$ The Authors. Published by Elsevier Inc. on behalf of The American Association for Thoracic Surgery. This is an open access article under the CC BY-NCND license (http://creativecommons.org/licenses/by-nc-nd/4.0/).

https://doi.org/10.1016/j.xjtc.2020.02.038
}

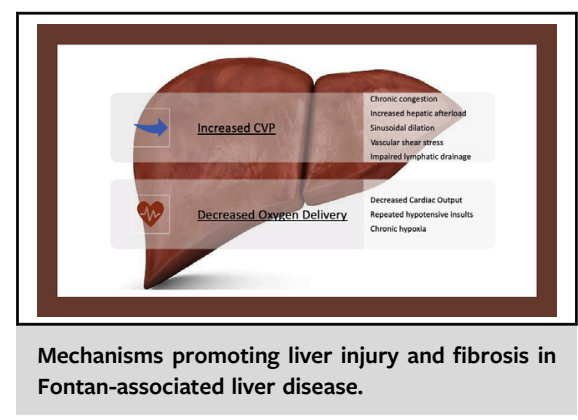

\section{CENTRAL MESSAGE \\ Hepatocellular carcinoma has a low prevalence in Fontan pa- tients, but it is a serious and potentially lethal complication. Further efforts are needed to systematize risk stratification and screening.}

disease. ${ }^{6}$ Among patients with liver cirrhosis, longitudinal studies suggest an annual risk of hepatocellular carcinoma (HCC) of $1 \%$ to $8 \%{ }^{7} \mathrm{HCC}$ is considered the most extreme manifestation of FALD. Most patients are asymptomatic and the diagnosis is usually incidental. The prognosis of $\mathrm{HCC}$ is very poor with survival rates estimated at $50 \%$ within 2 years of diagnosis. ${ }^{8,9}$

Wilson and colleagues ${ }^{10}$ describe a low prevalence (5 of 1620 patients) of HCC from the Australian and New Zealand Fontan Registry, which is among the largest series of Fontan patients with excellent short- and long-term outcomes. All patients in the registry who develop HCC had evidence of cirrhosis, portal hypertension, and history of failing Fontan circulation.

Although the reported prevalence of $\mathrm{HCC}$ in patients with Fontan circulation is low, there is currently no consensus on the optimal timing to initiate screening and imaging in post-Fontan populations. Patients with failing Fontan, portal hypertension, or evidence of liver cirrhosis are at higher risk of HCC. Surveillance for HCC includes serum tests (eg, total and direct bilirubin, aminotransaminases, alkaline phosphatase, gamma-glutamyl transferase, albumin, and international normalized ratio) and imaging. Post-Fontan patients with a confirmed diagnosis of cirrhosis should undergo more rigorous screening. Follow-up in these patients should include ultrasound and alphafetoprotein measurement. Immunization against hepatitis 


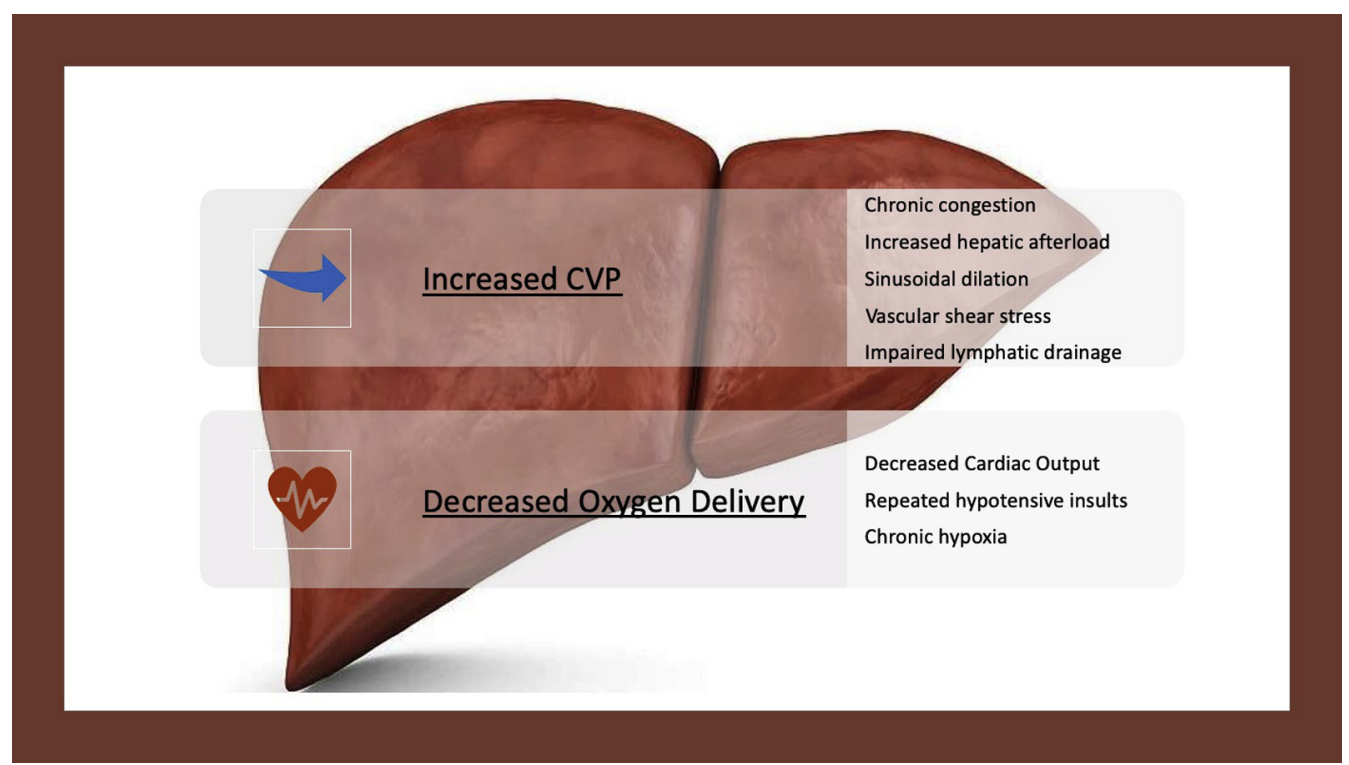

FIGURE 1. Mechanisms promoting liver injury and fibrosis in Fontan-associated liver disease.

A, hepatitis B, pneumococcal pneumonia, and influenza is also recommended. The use of pharmacologic agents such as pulmonary vasodilators (eg, sildenafil and bonsentan) and antifibrotic therapies could be considered to prevent the progression of FALD, although the effect on hepatic vascular resistance and on the prevalence of HCC is unknown and remains to be studied. ${ }^{4}$ Further efforts are needed to systematize risk stratification and screening guidelines in patients with FALD.

\section{References}

1. Rychik J, Atz AM, Celermajer DS, Deal BJ, Gatzoulis MA, Gewillig MH, et al. Evaluation and management of the child and adult with Fontan circulation: a scientific statement from the American Heart Association. Circulation. 2019. CIR0000000000000696.

2. d'Udekem Y, Iyengar AJ, Galati JC, Forsdick V, Weintraub RG, Wheaton GR, et al. Redefining expectations of long-term survival after the Fontan procedure: twenty-five years of follow-up from the entire population of Australia and New Zealand. Circulation. 2014;130:S32-8.
3. Gordon-Walker TT, Bove K, Veldtman G. Fontan-associated liver disease: a review. J Cardiol. 2019;74:223-32.

4. Kiesewetter CH, Sheron N, Vettukattill JJ, Hacking N, Stedman B, MillwardSadler H, et al. Hepatic changes in the failing Fontan circulation. Heart. 2007; 93:579-84.

5. Pundi K, Pundi KN, Kamath PS, Hacking N, Stedman B, Millward-Sadler H, et al. Liver disease in patients after the Fontan operation. Am J Cardiol. 2016; 117:456-60.

6. Nandwana SB, Olaiya B, Cox K, Sahu A, Mittal P. Abdominal imaging surveillance in adult patients after Fontan procedure: risk of chronic liver disease and hepatocellular carcinoma. Curr Probl Diagn Radiol. 2018;47: 19-22.

7. Ioannou GN, Splan MF, Weiss NS, McDonald GB, Beretta L, Lee SP. Incidence and predictors of hepatocellular carcinoma in patients with cirrhosis. Clin Gastroenterol Hepatol. 2007;5:938-45.

8. Egbe AC, Poterucha JT, Warnes CA, Connolly HM, Baskar S, Ginde S, et al. Hepatocellular carcinoma after Fontan operation: multicenter case series. Circulation. 2018;138:746-8.

9. Asrani SK, Warnes CA, Kamath PS. Hepatocellular carcinoma after the Fontan procedure. N Engl J Med. 2013;368:1756-7.

10. Wilson TG, Iyengar AJ, Hardikar W, Sood S, d'Udekem Y. Prevalence of hepatocellular carcinoma in the entire Fontan population of Australia and New Zealand. J Thorac Cardiovasc Surg Tech. 2020;2:128-30. 